\title{
Planning Velocity Profiles from Task-Level Constraints and Environment Uncertainties
}

\author{
Aleksandar Timcenko \\ Peter Allen \\ Center for Research in Intelligent Systems \\ Department of Computer Science \\ Columbia University, New York NY 10027 *
}

\begin{abstract}
This paper presents a method for parameterizing robot trajectories in the presence of uncertainties. We define the planning process as a problem of constrained optimization and use the concept of a task's difficulty as an optimization criterion. The task difficulty - as we define it - comprises the combined effects of velocity and uncertainty, mimicking human perception of difficulty in positioning tasks. The success probability is used as a constraint necessary for planning tasks with contradicting requirements.

We demonstrate this planning paradigm with an experiment that contains opposing requirements: reaching the obstacle in a given time, but without exceeding certain maximal impact force. We have implemented the planner on a real system.
\end{abstract}

\section{Introduction}

In this paper we are interested in defining methods for time parameterization of robot trajectories so that the robotic system achieves favorable performance despite the inevitable presence of uncertainties. In order to develop these methods, we need detailed understanding of the sources of random phenomena in the system, as well as comprehensive mathematical models of those phenomena. That understanding can lead us to a mathematically tractable formulation of motion planning in the form of a constrained optimization problem.

We will start with a very general definition of the planning problem based on the task's decomposition into groups of parameters. A robotic task can be characterized by a set of parameters. Here by "parameter" we mean both scalars, vectors, matrices and more complex mathematical object such as functions. The parameters can be grouped into several categories:

- controllable system parameters $\mathcal{P}_{\text {con }}$. Examples: nominal velocity, control structure gains. These parameters are at the designer's disposal to adjust and this "adjustment" if automatized is called "planning"

- known properties of the environment and the system that cannot be influenced $\mathcal{P}_{\text {env }}$. Example: geometrical constraints, probability distributions that describe sensor accuracy. These parameters are "the rules of the game". They cannot be changed

"This work was supported in part by DARPA contract DACA-76-92 C-007, NSF grants IRI-86-57151, CDA-90-24735, North American Philips Laboratories, Siemens Corporation and Rockwell International. by a system and they define the environment the system is acting in.

- constraints imposed on the task Preq. Examples: maximal allowed duration of a task, maximal allowed force exerted upon impact. These quantities define required system's performances.

- time $t$

The planning problem can be formulated as follows:

$$
\begin{aligned}
& \text { Given } \mathcal{P}_{\text {env }} \text { and } \mathcal{P}_{\text {req }} \text {, find } \mathcal{P}_{\text {con }} \text { that optimizes the cri- } \\
& \text { terion } \\
& \qquad \mathcal{J}\left(\mathcal{P}_{\text {con }}, \mathcal{P}_{\text {env }}, \mathcal{P}_{\text {req }}, t\right) \\
& \text { while maintaining the set of constraints } \\
& \qquad \mathcal{K}\left(\mathcal{P}_{\text {con }}, \mathcal{P}_{\text {onv }}, \mathcal{P}_{\text {req }}, t\right) \geq 0 \\
& \text { where } \mathcal{J} \text { is a given scalar and } \mathcal{K} \text { is a given vector function }
\end{aligned}
$$
in $\mathcal{P}_{\text {con }}, \mathcal{P}_{\text {env, }}, \mathcal{P}_{\text {req }}$ and $t$.

The real problem here is the choice of the criterion function $\mathcal{J}$ and the constraint function $\mathcal{K}$. We assume that once these functions are found, solving for optimal $\mathcal{P}_{\text {con }}$ can be conducted using appropriate numerical techniques. The main theme of this work is the choice of functions $\mathcal{J}$ and $\mathcal{K}$.

The examples of this planning framework are numerous. In robotics, there are several attempts to encompass motion planning into this framework. An example is the minimum-jerk velocity profile described in [7]. ${ }^{1}$ It can be briefly summarized as follows: rion

Find the velocity profile $\mathrm{q}(t)$ that minimizes the crite-

$$
\int_{0}^{T}\left(\frac{\mathrm{d}^{3} \mathbf{q}}{\mathrm{d} t^{3}}\right)^{2} \mathrm{~d} t
$$

if the system moves from a given point $\mathbf{q}_{A}$ to the given point $\mathrm{q}_{B}$ in time $T$.

Using the notation introduced above, this problem can be restated in the form

$$
\mathcal{J}=\min
$$

\footnotetext{
${ }^{1}$ This work offers a model of motion in living mechanisms (monkeys) but its applicability expands to their non-living counterparts.
} 
with

$$
\mathcal{K} \geq 0
$$

where $\mathcal{P}_{\text {con }}=\{\mathbf{q}\}, \mathcal{P}_{\text {env }}=\left\{\mathbf{q}_{A}, \mathbf{q}_{B}\right\}, \mathcal{P}_{\text {req }}=\{T\}, \mathcal{J}=$ $\int_{0}^{T}\left(\mathrm{~d}^{3} \mathbf{q} / \mathrm{d} t^{3}\right)^{2} \mathrm{~d} t$ and $\mathcal{K}=\left\|\int_{0}^{T} \mathbf{q} \mathrm{d} t\right\|-\left\|\overline{\mathbf{q}_{A} \mathbf{q}_{B}}\right\|$. In other words, the constraint $\mathcal{K} \geq 0$ guarantees that task's main objective is attained (sweep the distance between $q_{A}$ and $q_{B}$ in time $T$ ), while the criterion $\mathcal{J}=$ min ensures that it is being attained in some "preferable" way (in this case, with a minimal jerk on average).

Another attempt to encompass planning and control into a single concept is the potential-field method, originally described in [8]. If the configuration space was submerged into an electromagnetic field and if robot was a charged particle, we could expect it to obey to the laws goveming electromagnetic phenomena. Particularly, if the obstacles were sources of repulsive forces and the goal the source of attractive force, our particle-robot would eventually move towards the goal along the path that minimizes action. This force may be artificially introduced into a robot's dynamic equations, yielding its behavior similar to the one of the charged particle in the electrostatic field. As stated in [9], the trajectories that originate close to the minimum of the potential field $U_{\text {ext }}$ will eventually converge to that minimum.

The potential field planning paradigm is related to the optimal kinodynamic planning [4] where the goal is to plan the trajectories that are time-optimal and that satisfy certain dynamic constraints. The analysis of the complexity of finding optimal plans and their "good" approximations is given in [3] and related papers. It has been shown that the optimal plans are NP-hard to find, but the "good" approximations can be found in polynomial time.

Uncertainty introduces further difficulties. The planning problem gets severely more complicated if the system cannot guarantee that the point that it needs to reach is exactiy at $q_{B}$. If that is an object that should be grasped, its position may not be - and, as a matter of fact, never is - exactly known to the system. Thus, we need a plan that will answer a question like "What is the velocity profile that most probably results in reaching the object while maintaining the constraints such as maximal acceleration and maximal jerk in prescribed boundaries?". This is the question we want to address in this research. More precisely, we pose the following problem:

A system's knowledge about itself and the environment is given by the vector $\mathcal{P}_{\text {env }}$ (elements of $\mathcal{P}_{\text {env }}$ can be either parameters or parameters' probability distributions). The requirements that the task has to meet are given by the vector $\mathcal{P}_{\text {req. }}$. Find the criterion $\mathcal{J}$ and the set of constraints $\mathcal{K}$ and solve the constrained optimization problem

$$
\left\{\begin{array}{l}
\mathcal{J}=\min \\
\mathcal{K} \geq 0
\end{array}\right.
$$

in the vector of controllable parameters $\mathcal{P}_{\text {con }}$ so that the re sulting system's behavior has certain intuitively favorable properties.

Before we elaborate on this formulation, let's make a quick comment on how mother nature deals with a similar problem. It has been recognized in the psychology literature (see for example [7, 2]) that the time-constrained pointing movements of monkeys as well as humans follow a bell-shaped profile that is close to a solution of a minimum-jerk model. However, if the goal accuracy demands are increased, the velocity profile tends to "skew" [6], demonstrating slower and more careful approach to the goal, the phenomenon not predicted by criterion 1 . That implies that the required accuracy (or, in other words, allowed error) should be encoded into the criterion. We know that it is more difficult to move fast, as well as to position accurately. The criterion of the form 1 constrains only the velocity while leaving the accuracy untouched. Intuitively, a better criterion would have the form

$$
\left.\int_{0}^{T} \text { (velocity }+ \text { accuracy }\right) \mathrm{d} t=\min
$$

where velocity and accuracy are functions of appropriate form that reffect the required velocity and accuracy. Our goal is to formulate such a criterion, justify it and ultimately apply it in solving the planning problem. We will call the criterion of the form 3 a task's difficulty inde $x^{2}$ and denote it by $\mathcal{D}$.

Now let's go back to the formulation of the planning problem presented above. The phrase "intuitively favorable properties" needs further explanation. The criterion, as well as the set of constraints, reflects the desired behavior of the system in the mathematical form. The representation of our desires in the mathematical form inevitably introduces approximations, simplifications and modeling errors. If the task our robotic system is supposed to undertake is, say, grasping an object laying on a desk, the "intuitively favorable property" of a plan for that task would be that most of the time the robot actually grasps the given object. In mathematical terms, that would mean that the requirement imposed on the planner is that the probability of the successful performance should be above a certain threshold. Thus, in order to state the problem, a human planner is supposed to choose the "intuitively favorable property" (such as success probability), write it down mathematically, and then let computer planner optimize it.

The formulation of the planning problem as stated in 2 is too general; we will confine our scope to the special case when $\mathcal{P}_{\text {env }}=$ $\left\{\psi_{\mathbf{q}}\right\}$ (the environment is described by a probability distribution function in the configuration space) and $\mathcal{P}_{\text {con }}=\{\mathbf{q}\}$ (planning result is a velocity profile). The set of requirements will vary; some of the requirements that we have considered are expected completion time and the expected impact force upon the collision with an obstacle. In section 3 we will review step-by-step the planning process and try to point to possible problems on the way.

\section{The Review of the Continuous Uncertainty Model}

For modeling random phenomena present in the robotic system we will use the continuous uncertainty model, presented in greater details in $[10,11]$. Here we will give a brief overview.

There are three main types of uncertainties present in the robotic system: sensor, control and environment uncertainty. Motion of the system in the presence of those uncertainties is given by the stochastic system

$$
\mathrm{d} \mathbf{q}_{t}^{w}=d \mathbf{q}_{t}^{m}+\Sigma^{w}\left(\mathbf{q}_{t}^{m}\right) \mathrm{d} W^{w}
$$

\footnotetext{
${ }^{2}$ This term is not new; it exists in the psychology literature for almost 40 years (see [5]).
} 


$$
\begin{aligned}
\mathrm{d} \mathbf{q}_{t}^{m} & =\mathrm{d} \mathbf{q}_{t}^{c}+\Sigma^{m} \mathrm{~d} W^{m} \\
\mathrm{~d} \mathbf{q}_{t}^{c} & =\mathrm{d} \mathbf{q}_{0}^{c}=\mathbf{v}^{c} \mathrm{~d} t
\end{aligned}
$$

with the initial conditions

$$
\begin{aligned}
\mathbf{q}_{0}^{c}=\mathbf{q}_{0}^{m} & \sim \mathcal{N}\left(\mathbf{q}_{0}^{0}, \Sigma^{c}\right) \\
\mathbf{q}_{0}^{w} & \sim \mathcal{N}\left(\mathbf{q}_{0}^{0}, \Sigma_{0}^{\omega}\right)
\end{aligned}
$$

where

- $\mathbf{q}_{0}^{0}, \mathbf{q}_{t}^{0}$ are the nominal positions in time instances 0 and $t$ (under the assumption that there are no uncertainties in the system)

- $\mathbf{q}_{0}^{c}, \mathbf{q}_{t}^{c}$ are the positions that would be retrieved by a sensor system in time instances 0 and $t$ under the nominal (commanded) velocity (i.e. under the assumption that the only uncertainty present in the system is the sensor uncertainty)

- $\mathbf{v}^{c}$ is the nominal (commanded) velocity

- $\mathrm{q}_{0}^{m}, \mathbf{q}_{t}^{m}$ are the effective positions in time instances 0 and $t$ (robot positions as a result of sensor and control uncertainties combined)

- $\mathbf{q}_{t}^{w}$ is the actual position (the combination of all three uncertainties)

Thus, the overall uncertainty model is defined by three constant quantities $\left(\Sigma^{m}, \Sigma^{c}, \Sigma_{0}^{w}\right)$ and one function that describes the environment uncertainty $\left(\Sigma^{w}\right)$. A point in the configuration space is thus represented by a random vector with Gaussian distribution. We call this model "the continuous uncertainty model".

\section{The Task Difficulty as an Optimization Criterion}

The valid question that can be asked about the concept of task difficulty is if there is a quantity that numerically reflects the intuitive notion of difficulty (as human's perceive it) in the first place. What we are searching for at this point is a definition (which may not be unique) that does not violate our intuitive expectations on some simple examples (such as positioning and reaching), and that can be used as a criterion in planning. The need for such a criterion exists and our understanding is that it should satisfy the conditions presented above.

The freedom in choosing a definition for the difficulty index should be bounded by certain intuitive properties difficulty index should possess (as elsewhere, $\mathcal{D}$ stands for difficulty index):

- $\mathcal{D}$ increases as average velocity, acceleration or jerk increase

- $\mathcal{D}$ increases as the amount of uncertainty increases

- $\mathcal{D}$ is additive, meaning that the difficulty $\mathcal{D}(T)$ of a task $T$ that consists of two subtasks $T_{1}$ and $T_{2}$ performed in a sequence is the sum of particular difficulties:

$$
\mathcal{D}(T)=\mathcal{D}\left(T_{1}\right)+\mathcal{D}\left(T_{2}\right)
$$

- $\mathcal{D}$ depends only on dynamic properties of a task. In other words, it is invariant of the position in the configuration space (it does not matter where it is performed), as well as of the sensor uncertainty. This last condition means that no matter how (in)accurate the sensors are, task's difficulty shouldn't depend on that - no matter what we do, system's accuracy will be bounded below the sensor's accuracy.
One candidate for $\mathcal{D}$ is the integral of the form

$$
\mathcal{D}=\int_{0}^{T}\left(\left(\frac{\mathrm{d}}{\mathrm{d} t} \mathbf{E} \mathbf{q}_{t}^{w}\right)^{2}+\frac{\mathrm{d}^{2}}{\mathrm{~d} t^{2}}\left(\mathbf{E} \mathbf{q}_{t}^{w 2}-\left(\mathbf{E} \mathbf{q}_{t}^{w}\right)^{2}\right)\right) \mathrm{d} t
$$

where $\mathbf{E}$ stands for the expectation operator. This formula reads: the difficulty is the integral of the sum of the expected velocity squared and second derivative of the expected variance. If we assume constant environment uncertainty $\Sigma^{w}$ (as we will in the next section), it turns out that $\mathcal{D}$ becomes:

$$
\mathcal{D}=\int_{0}^{T}\left(\mathbf{q}_{t}^{w 2}+\Sigma^{m} \ddot{\mathbf{q}}_{t}^{w}\right) \mathrm{d} t
$$

This form clearly resembles the desired form for the criterion expressed in 3: the combination of velocity and uncertainty constraints. Other desirable properties of $\mathcal{D}$, listed above, are easily verified.

\section{Planning of a Velocity Profile under Uncertainty}

In this section, we will describe the application of our method to planning velocity profiles for constrained motion amidst obstacles. In particular, this method allows us to compute a difficulty index as defined in 4 that we can use as an optimization criteria for planning a velocity profile in a cluttered and uncertain environment. The constraint we impose on the planner is that a success probability is above certain threshold.

Let us define the following binary events for each time instant (these will allow us to cast this task as a compound binary predicate):

$$
\begin{aligned}
& S=\text { (success) } \\
& T T=\text { (elapsed time before impact } \leq \tau_{\max } \text { ) } \\
& \left.F=\text { (impact force } \leq f_{\max }\right) \\
& I M=\text { (impact has occurred) }
\end{aligned}
$$

We will define the probability of success, $\Psi\{S\}$, as an intersection of two events: getting to the goal in time, and not exceeding the maximal force:

$$
\Psi\{S\}=\Psi\{T T \cap F\}
$$

Assuming that $T T$ and $F$ are mutually independent, we have

$$
\Psi\{S\}=\Psi\{T T\} \Psi\{F\}
$$

Now we compute $\Psi\{F\}$. Applying simple set algebra we have the following relations:

$$
\begin{aligned}
& \Psi\{F\}=\Psi\{F \cap I M \cup F \cap \overline{I M}\} \\
& (F \cap I M) \cap(F \cap \overline{I M})=\emptyset \Rightarrow \\
& \Psi\{F \cap I M \cup F \cap \overline{I M}\}=\Psi\{F \cap I M\}+\Psi\{F \cap \overline{I M}\} \\
& \Psi\{F \cap \overline{I M}\}=\Psi\{F \mid \overline{I M}\} \Psi\{\overline{I M}\}
\end{aligned}
$$


Since $\Psi\{F \mid \overline{T M}\}=1$ (the probability of not exceeding the force under the assumption that the impact has not occurred is 1 - the obstacle is simply not reached yet):

$$
\Psi\{F \cap \overline{I M}\}=\Psi\{\bar{I} \bar{M}\}
$$

Therefore,

$$
\begin{aligned}
\Psi\{F\} & =\Psi\{F \cap I M\}+\Psi\{\overline{I M}\} \\
& =\Psi\{F \mid I M\} \Psi\{I M\}+1-\Psi\{I M\}
\end{aligned}
$$

and finally:

$$
\Psi\{F\}=1-\Psi\{I M\}(1-\Psi\{F \mid I M\})
$$

Thus we have written the success probability $\Psi\{S\}$ as a function of three elementary probabilities: $\Psi\{T T\}, \Psi\{I M\}$ and $\Psi\{F \mid I M\}$, that can be computed from the uncertainty model in section 2 . The probabilities $\Psi\{T T\}, \Psi\{I M\}$ and $\Psi\{F \mid I M\}$ can be computed from the uncertainty model (See Appendix A).

Let us consider a task of moving along the prescribed path unti colliding with an obstacle, and then exerting a prescribed force on the surface of the obstacle. It consists of three phases: moving along the path, colliding, and maintaining the prescribed force. The problem encountered in practice is a manipulator's tendency to bounce from the surface upon initial collision, especially in the case of a very rigid obstacle. It has been shown [1] that simple spring control cannot successfully cope with that problem. Essentially, it is caused by the necessity to instantly change the characteristics of motion; in our example to stop and exert a force. Since the manipulator system is not capable of stopping instantly after collision due to its internal delays, it bounces and approaches the obstacle again. If the spring constant is high enough it will bounce again and keep doing that forever. That required instantaneousness is the core of the problem: something has to be rapidly changed, and the system might not be able to perform that.

The system's knowledge about the environment is based on models provided by a programmer, and those models are obtained by quantitatively describing the positions and dimensions, as well as other characteristics of objects which constitute the environment. The more accurate those models are, the system can - at least theoretically - utilize that knowledge more efficiently in order to attain the goal of the task more accurately. In our experiment, if the knowledge of the environment is exact, that is, if the position and the elasticity coefficient of the obstacle are known, the system could move with the maximum speed to the point of contact, computed such that it inflicts the elastic deformation of the obstacle proportional to the required force. On the other extreme, if the knowledge about the environment is zero, the system has to slowly wander through the darkness until it encounters the obstacle, and then to utilize a certain control scheme for maintaining a given force, based on force measurements.

The reality is somewhere in between. The knowledge which is at the system's disposal may be substantial, yet not enough to guarantee that the "full knowledge" strategy is a reasonable choice. We may assume that it is quite unlikely that the obstacle is in a certain region, thus allowing the robot to pass through that region swiftly, while slowing down in the region where the obstacle is expected to be. That means that parameters we can control (velocity in this example) depend on the overall uncertainty of the system and the environment.
So, given a velocity, we can compute the probability that the system will fulfill a task within a pre-determined set of constraints such as maximum time for the total motion and maximal impact force upon contact. Our method is to find a velocity at each step of the motion that maximizes the success probability (defined below) and link these into a overall velocity profile for the task given the constraints.

The experiment that we have conducted to demonstrate the use of a success probability in velocity profile planning consists of moving until reaching an obstacle, and exerting a given force after the impact. Let us impose two requirements on our system: the total elapsed time of motion before the impact should be at most $\tau_{\max }$, and the maximal force exerted upon contact should not exceed $f_{\max }$. Those two requirements are contradictory: while the former requires the velocity to be high, the latter pushes it back.

Figure 1 shows the planning of the velocity profile through the interplay of the task difficulty (equation 4) and success probability (equation 5 from the Appendix). In accordance to 2, the constrained optimization problem is given by the system

$$
\left\{\begin{array}{l}
\mathcal{D}=\min \\
\Psi\{S\} \geq \Psi_{0}
\end{array}\right.
$$

The axis denoted $z$ is the distance along $z$ coordinate. That is the direction of motion in which the obstacle is positioned. The axis denoted $z^{\prime}$ is the velocity along $z$ coordinate. The task's difficulty is shown on the vertical axis, and the success probability by gray shades. Lighter shades stand for high success probabilities. Both task the difficulty and success probability are functions of the velocity profile. By considering the model of uncertainty and relevant environmentparameters such as obstacle's elasticity properties (elasticity coefficient Eh) and the inherent system delay (pDelay, expressed in sampling intervals), planner estimates both the motion duration and impact force and the probabilities that they will both stay inside prescribed boundaries. The parameter sigma is the measure of environment uncertainty $\Sigma^{m}$. Higher values of $\Sigma^{m}$ cause the system to be more "cautious", slowing down further from the obstacle. Other relevant parameters are shown as well: the requested maximal motion duration ( $\mathrm{tau}$ ), sampling interval $\mathrm{T}$, and requested maximal impact force $\mathrm{fm}$. The planned trajectory is shown by a solid line. It demonstrates the planner's tendency to "thread" a trajectory through the areas of high success probability.

Using the model shown in figure 1 , we have planned a trajectory that has optimized the success probability for the impact task. Figures 2,3 and 4 are the actual data recorded from a PUMA-560 with wrist sensor that was given a certain motion duration $\left(\leq \tau_{\max }\right.$ ), and impact force to be minimized ( $\leq f_{\max }$ ), in the presence of the environment uncertainty. Figure 2 is the force measured before and after impact. It shows that the maximal requested force has not been exceeded. Figure 3 is the time diagram of robot's position during the approach and figure 4 is the phase space (position $\times$ velocity) trajectory, analogous to one in figure 1 . The velocity profile that maximizes the success probability has the shape that one would intuitively expect: in the area where the obstacle is unlikely to be, the robot starts with a high negative velocity (negative velocity since the direction of movement is downward) and then slows down in order to have a controlled impact force upon the collision. Thus, we can precompute velocity profiles using our model that are able to be mapped into actual robot control strategies. 


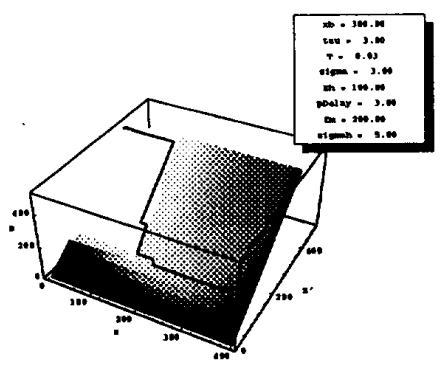

Figure 1: Modeled trajectory using the uncertainty model. Darker areas mean a low probability of success, lighter mean a higher probability of success. For example, low velocities will not allow task completion in the required elapsed time $\left(\leq \tau_{\max }\right)$. High velocities may cause impact greater than $f_{\max }$.



Figure 2: The force before and after impact. The horizontal axis is the time in milliseconds and the vertical axis is the force in Newtons.



Figure 3: Trajectory time diagram. Horizontal axis is the time in milliseconds and vertical the obstacle position in $\mathbf{m m}$.
Figures 5-8 show planning results under different extreme circumstances. Figure 5 shows that if the allowed impact force is high ( $\mathrm{fm}$ $=10000$ ) the system will move with maximal velocity. Figure 6 shows the trajectory in the case of very low uncertainty ( $\operatorname{sigma}=$ 0.01 ). System moves directly to the goal and then slows down. The success probability is high. Figure 7 shows the opposite extreme: the planning under very high uncertainty $(\mathrm{sigma}=5)$. Since the obstacle position is virtually unknown and the time constraint is tight, the success probability is low. However, if we relax the time constraint $(\mathrm{tau}=10)$, even in the case of high uncertainty we can achieve high success probability. The trajectory is shown in figure 8 .

\section{Conclusion}

In this paper we have implemented the concept of a difficulty index that combines velocity and force constraints along with the inherent uncertainty present in the system. From this we have created the optimization criterion and used it together with a success probability in planning robot trajectories. We have outlined some of the conditions a "good" difficulty index should satisfy, and proposed one that we have used in the planning of an actual trajectory that incorporates time and force constraints to compute the effective velocity. This work is part of a broader effort to combine into a comprehensive system problems of path and trajectory planning in the presence of uncertainties.

We believe that this is a fruitful research direction. It opens a wide spectrum of questions. Some of them are: 1) dealing with non-constant environment uncertainty, 2) the robustness of obtained plans with respect to modeling errors, 3 ) the numerical complexity of computing approximations of globally optimal plans, 4) experimentation with different types of difficulty indices and different types of constraint functions, such as mathematical expectations instead of success probabilities. We hope to address some of these problems in future.

\section{References}

[1] C. H. An and J. M. Hollerbach. The role of dynamic models in cartesian force control of manipulators. International Journal of Robotics Research, 8(4), 1989.

[2] C. G. Atkeson and J. M. Hollerbach. Kinematic features of unrestrained vertical arm movements. The Journal of Neuroscience, 5(9):2318-2330, 1985.

[3] J. Canny, B. Donald, J. Reif, and P. Xavier. On the complexity of kinodynamic planning. In 29 th Symposium on the Foundations of Computer Science, 1988.

[4] B. Donald and P. Xavier. A provably good approximation algorithm for optimal-time trajectory planning. In Proceedings of the IEEE Conference on Robotics and Automation, 1989.

[5] P. M. Fitts. The information capacity of the human motor system in controlling the amplitude of the movement. Journal of Experimental Psychology, 47:381-391, 1954.

[6] B. Hoff and M. A. Arbib. A model of the effects of speed, accuracy and perturbation on visually guided reaching. In $\mathrm{R}$. Caminiti, editor, Control of Arm Movements in Space: Neurophysiological and Computational Approaches. Springer-Verlag, 1991.

[7] N. Hogan. Organizing principle for a class of volyntary movements. The Journal of Neuroscience, 4:2745-2754, 1984.

[8] $O$. Khatib. Real-time obstacle avoidance for manipulators and mobile robots. International Journal of Robotics Research, 5(1):90-98, 1986.

[9] D. E. Koditschek. Robot planning and control via potential functions. In O. Khatib, J. J. Craig, and T. Lozano-Pérez, editors, The Robotics Review 1. MIT Press, 1989. 


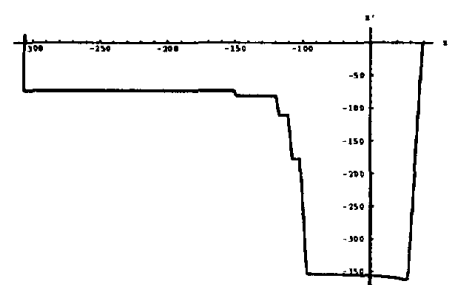

Figure 4: The velocity profile planned so that the motion duration and the impact force are minimized at the same time. The horizontal axis is the position and the vertical axis is the velocity. Note that the motion is "downwards", thus we have negative velocity.

[10] A. Timcenko and P. Allen. Modeling uncertainties in robot motions. In Applications of AI to Real-World Autonomous Mobile Robots, AAAI Fall Symposium Series, 1992.

[11] A. Timcenko and P. Allen. Modeling dynamic uncertainty in robot motion. In Proc. IEEE International Conf. on Robotics and Automation, 1993.

\section{A Derivation of probabilities $\Psi\{T T\}$ and $\Psi\{I M\}$}

Let us assume that the obstacle is positioned at $\overline{q^{w}}$, and that the current time instant is $t$, so that the robot's position is $\mathbf{q}_{t}^{w}$. We clearly have $\overline{\mathbf{q}^{w \prime}}>\mathbf{q}_{t}^{t \omega}$ since the impact hasn't occurred yet. The probability that the obstacle will be reached in the remaining time $\tau_{\max }-t$ is

$$
\Psi\{T T\}=\Psi\left\{\int_{\mathbf{q}_{i}^{w}}^{\overline{\mathbf{q}^{w}}} \frac{\mathrm{d} \eta}{v(\eta)}<\tau_{\max }-t \mid \overline{\mathbf{q}^{w}}>\mathbf{q}_{m}^{w}\right\}
$$

From the uncertainty model we can compute probability density functions $\psi_{\overline{\mathbf{q}^{\boldsymbol{w}}}}$ and $\psi_{\mathbf{q}_{t}^{w}}$ of random variables $\overline{\mathbf{q}^{w}}$ and $\mathbf{q}_{t}^{w}$, respectively. Substitution in 6 results in the expression for the probability $\Psi\{T T\}$ :

$$
\Psi\{T T\}=\int_{-\infty}^{\infty} \psi_{\mathbf{q}_{t}{ }^{\omega}}\left(\mathbf{q}_{1}\right) \mathrm{d} \mathbf{q}_{1} \int_{\mathbf{q}_{1}}^{\mathbf{q}_{1}+\int_{t}^{\tau_{\max }} v \mathrm{~d}_{t}} \psi_{\overline{\mathbf{q}^{\omega}}}\left(\mathbf{q}_{2}\right) \mathrm{d}_{\mathbf{q}_{2}}
$$

( $\boldsymbol{q}_{1}$ and $\boldsymbol{q}_{2}$ are integration variables).

The impact probability $\Psi\{I M\}$ can be computed similarly from

$$
\Psi\{I M\}=\Psi\left\{\mathbf{q}_{t}^{w}+\int_{t}^{t+T} v \mathrm{~d} t>\overline{\mathbf{q}^{w}} \mid \mathbf{q}_{t}^{w}<\overline{\mathbf{q}^{w}}\right\}
$$

i.e. it is the probability that the impact occurs during the next sampling interval $T$, under the assumption that it hasn't occurred yet.

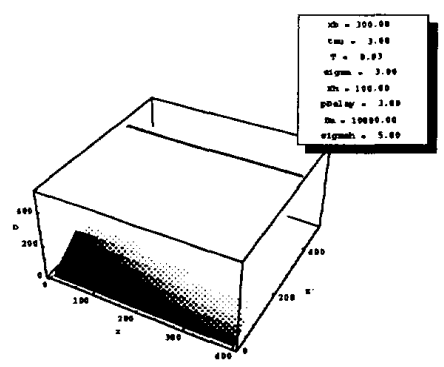

Figure 5: High impact force allowed



Figure 6: Low uncertainty

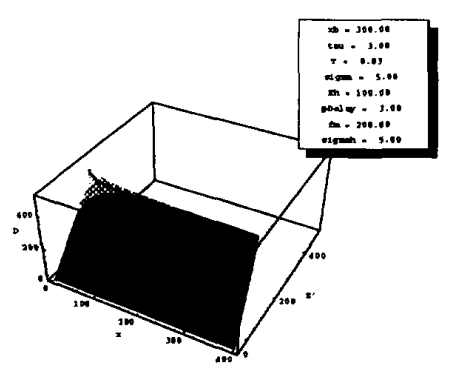

Figure 7: High uncertainty

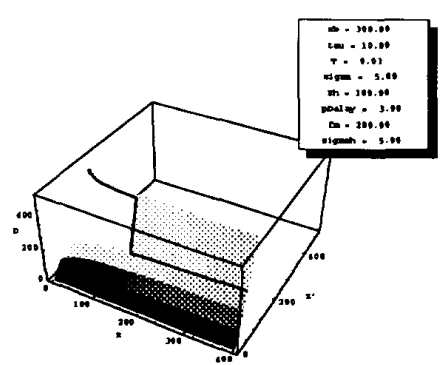

Figure 8: High uncertainty, long motion time allowed 